\title{
Neutrinos from Cosmic Ray Interactions in the Sun as background for dark matter searches
}

\author{
M. Ardid ${ }^{a}$, I. Felis ${ }^{a}, M$. Lotze $^{b}$ and C. Tönnis ${ }^{b, 1}$, on behalf of the ANTARES \\ Collaboration \\ ${ }^{a}$ Institut d'Investigació per a la Gestió Integrada de les Zones Costaneres (IGIC) - Universitat \\ Politècnica de València. C/ Paranimf 1, 46730 Gandia, Spain. \\ ${ }^{b} I F I C$ - Instituto de Física Corpuscular, CSIC - Universitat de València. C/ Catedrático José Beltrán 2, \\ E-46980 Paterna, Valencia, Spain \\ E-mail: mardid@fis.upv.es, ivfeen@upv.es, moritz.lotzelific.uv.es, \\ ctoenniseific.uv.es
}

\begin{abstract}
Neutrino telescopes have been proposed as efficient tools for indirect dark matter searches, especially using the Sun as source for its good capability to capture dark matter and since we do not expect high-energy neutrinos from it. However, the last statement should be taken with caution because high-energy neutrinos may come from cosmic particle interactions in the atmosphere of the Sun and producing neutrinos. In this work, we describe an analysis of the ANTARES neutrino telescope optimised for the observation of neutrinos coming from the atmosphere of the Sun due to cosmic particles interactions. Focusing in the $10 \mathrm{GeV}-10 \mathrm{TeV}$ energy range and using 2007-2012 data, the sensitivity obtained for the flux is approximately $10^{12} \mathrm{~km}^{-2} \mathrm{y}^{-1}$, whereas the expected flux is two order of magnitudes below. From this, we can conclude that present high-energy neutrino telescopes dark matter searches in the Sun can indeed neglect this contribution, but could play a role in future detectors with better neutrino flux sensitivities in the $10 \mathrm{GeV}-10 \mathrm{TeV}$ energy range and very good angular resolution.
\end{abstract}

\footnotetext{
${ }^{1}$ Speaker.
} 


\section{Introduction}

There are several techniques to detect high energy neutrinos, but for the energy range of interest between $10^{10}$ and $10^{16} \mathrm{eV}$, the most exploited method is the detection with large volumes of dense material (such as water or ice) by photomultipliers sensitive to Cherenkov light. As neutrinos can interact with atomic nuclei to produce charged leptons that emit Cherenkov radiation in water, this optical pattern can be used to infer direction, energy, and more information about incident neutrinos. This is the technique used by the ANTARES underwater neutrino telescope [1]. Data from this detector have been used in different Dark Matter (DM) searches in the Sun [2] [3] [4]. In this kind of searches the neutrino flux produced by cosmic rays interacting with the atmosphere in the Sun is usually neglected. In this paper, we use the ANTARES detector to study this flux and to derive possible consequences for DM searches in the Sun through neutrino detection.

Cosmic ray impingement on the solar atmosphere leads to the production of secondary particles via high energy pp-interactions, the decay of which results in the flux of both electron and muon neutrinos and antineutrinos.

Studies of the flux of neutrinos originating from cosmic ray interactions with matter in the Sun have been performed with Monte Carlo models for high energy particle interactions [5] [6] [7] [8] [9]. To do this, it has been taken into account the interplanetary solar magnetic fields and the shadowing effect of inelastic neutrino scattering in the Sun. The Solar Atmosphere Neutrino $(\mathrm{SAv})$ spectra may be altered by neutrino oscillations, which depend on the neutrino mass differences and mixing matrices. The resulting flux at the Earth (within the Sun's solid angle) is higher than the corresponding one from cosmic ray interactions with the Earth atmosphere, so it is a potential source of background for dark matter searches based on detection of neutrinos coming from the Sun.

\section{Expected neutrino fluxes from the Sun}

In recent years, significant improvements have been made in the modelling of the solar atmosphere. Ingelman and Thunman [10] used a semi-empirical 1D model for solar density from the data of [11] for the atmosphere (updated in [12]) and [13] for the deeper layers of the Sun. It can be parameterized using the following expression: $(h)=\rho_{0} e^{-h / h_{0}}$, where $h>0$ and $h<0$ are the locations above and below the solar radius $R_{\odot}$, respectively. The parameters $\rho_{0}$ and $h_{0}$ are presented in Table 1 .

\begin{tabular}{ccc}
\hline$h[\mathrm{~km}]$ & $\rho_{0}\left[\mathrm{~g} / \mathrm{cm}^{3}\right]$ & $h_{0}[\mathrm{~km}]$ \\
\hline$h>0$ & $3.68 \cdot 10^{-7}$ & 115 \\
\hline$-2000<h<0$ & $3.68 \cdot 10^{-7}$ & 622 \\
\hline$h<-2000$ & $45.3 \cdot 10^{-7}$ & 2835 \\
\hline
\end{tabular}

Table 1. Parameters that define the profile of solar density.

The most current profiles [6] [7] start from this 1D model and complement it with additional requirements. In Figure 1 we show an outline of how the particles travel through the Sun, as well as the density profile. The incoming cosmic rays interact with the Sun creating secondary particles that interact or decay producing neutrinos. It is observed that, depending on 
the angle of impact with respect to the axis connecting the Sun and Earth, the interaction length varies, being greater for smaller angles.

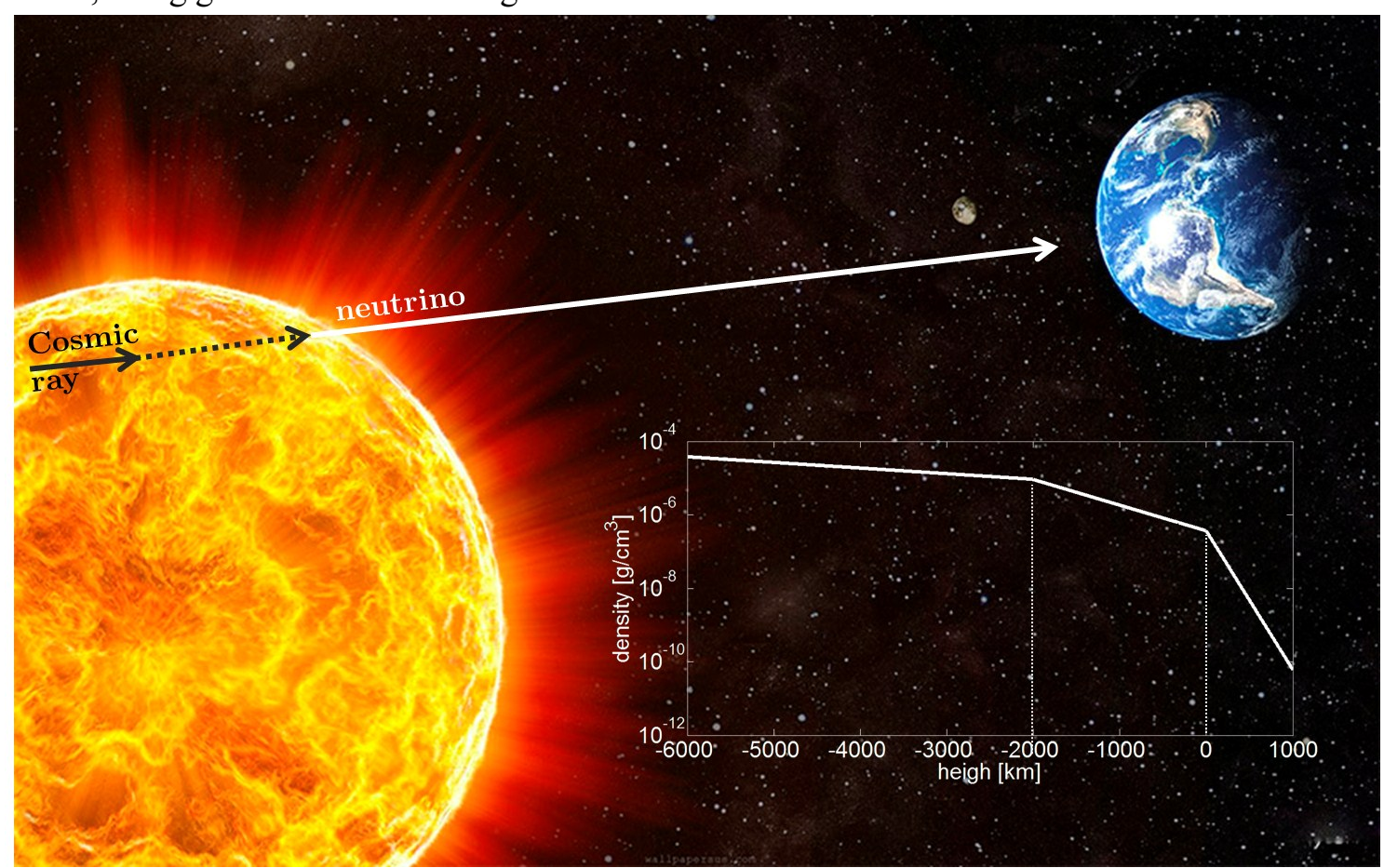

Figure 1. Neutrino production scheme of the solar atmosphere and density profile of the Sun.

In Figure 2 we show a first approximation of the expected SAv flux in the Earth without considering the oscillations (left) and considering the oscillations (right) [5]. For comparison, the flux of neutrinos from the Earth atmosphere is also shown on the left plot. We can infer that, for the energy range of interest $(10 \mathrm{GeV}-10 \mathrm{TeV})$ the total expected flux is approximately $2.7 \cdot 10^{10} \mathrm{~km}^{-2} \mathrm{y}^{-1}$.

Without oscillations

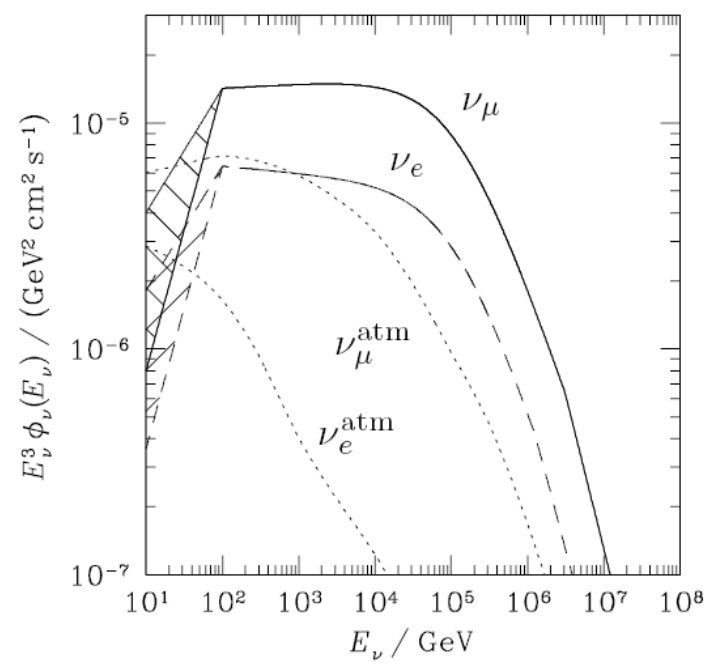

With oscillations

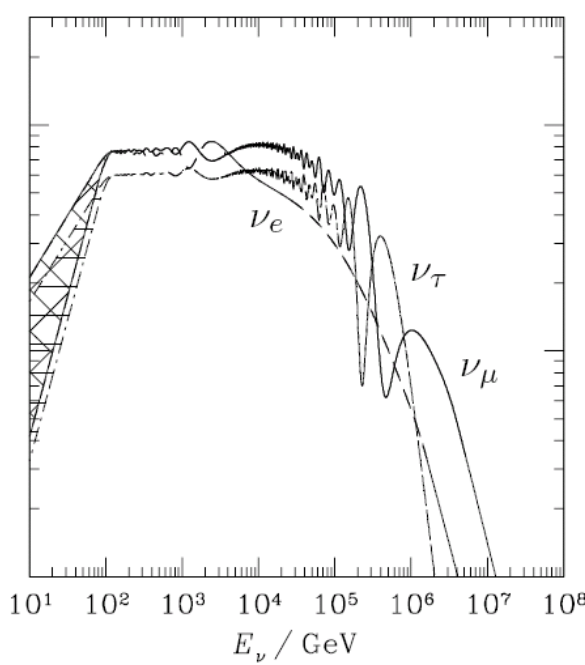

Figure 2. Comparison of expected atmospheric solar neutrino fluxes on Earth, with and without oscillation, for the three neutrino families. From [5]. 
From most recent studies [6] we extract the fluxes (Figure 3) from each of the neutrino and antineutrino flavours of the solar atmosphere that reach the Earth (solid line), compared to those produced in the solar atmosphere (discontinuous). Shaded bands show the region of uncertainty in all models. In this case, we have the dependence on the angle with respect to the solar centre. These fluxes have been used in the studies.

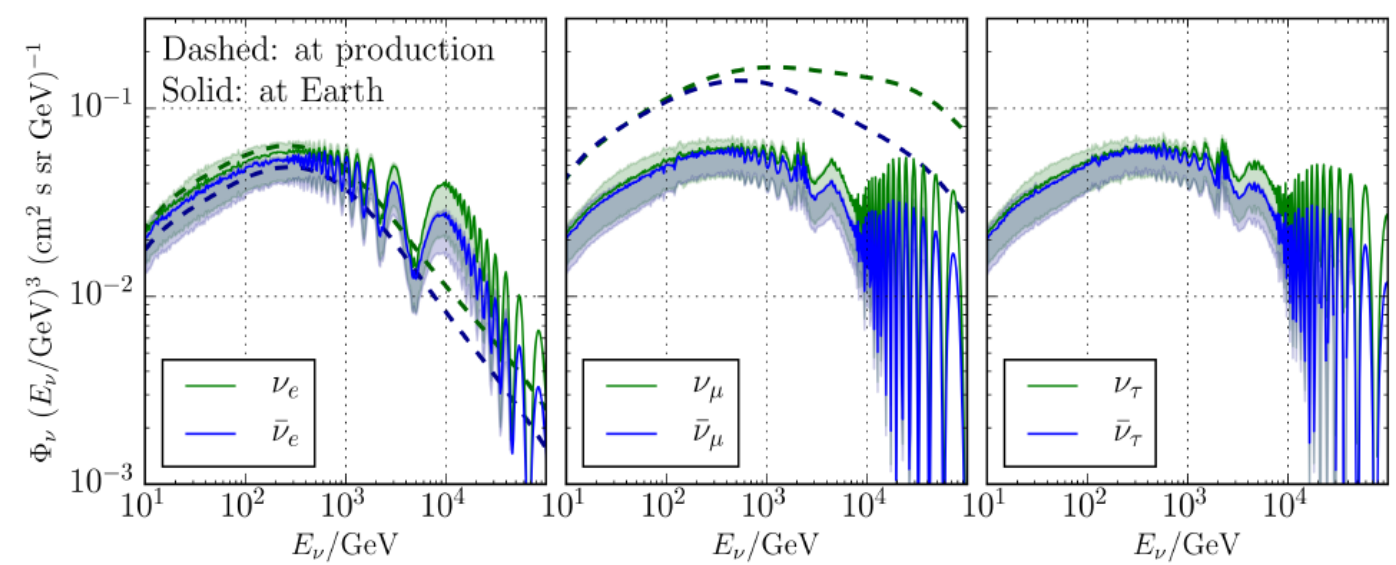

Figure 3. Fluxes of the three neutrino and antineutrino flavours at production in the atmosphere of the Sun and on Earth. From [6].

\section{ANTARES search for neutrinos from cosmic ray interactions in the Sun}

To place the most restrictive limits on a signal model, the strategy to choose the appropriate event selection is based on two steps:

$1^{\text {st }}$ Step. From a flux of particles known by a physical model of a source (e.g. the Sun) and the efficiency of the telescope (expressed through the effective area), we can obtain the sensitivity to that flux from a model of rejection. For this, the parametric cuts that optimize the sensitivity to this flux are looked for. Although we cannot know the actual upper limit that will result from an experiment until we see the data, we can use in this step the Monte Carlo predictions to calculate the sensitivity (average upper limit calculated according to FeldmanCousins [14]) that would be observed after a hypothetical repetition of the experiments with an expected background, $n_{b}$, and no true signal $\left(n_{s}=0\right)$. This average upper limit, $\bar{\mu}_{90}$, is the sum of the expected upper limits, $\mu_{90}\left(n_{o b s}, n_{b}\right)$, weighted by its probability of occurrence of Poisson, i.e.:

$$
\bar{\mu}_{90}\left(n_{b}\right)=\sum_{n_{o b s}}^{\infty} \mu_{90}\left(n_{o b s}, n_{b}\right) \frac{\left(n_{b}\right)^{n_{o b s}}}{\left(n_{o b s}\right) !} e^{-n_{b}}
$$

On a set of identical experiments, the strongest constraint on the expected flow of the signal $\Phi$ corresponds to the set of selection cuts that minimizes the model rejection factor and, therefore, minimizes the upper limit of average flow that would be obtained on the hypothetical experimental set. For the purposes of calculation with neutrino telescopes, we will use the following expression [15] [2]: 


$$
\bar{S}\left(n_{b}\right)=\Phi \frac{\bar{\mu}_{90}}{n_{s}}=\frac{\bar{\mu}_{90}}{A_{E f f} \cdot T}
$$

where $A_{E f f}$ is the average effective area in the energetic range in question for both neutrinos and antineutrinos and $T$ is the live time of the detector.

$2^{\text {nd }}$ Step. With the values obtained in the first step, the flux observed by the telescope is searched applying these selection cuts and if no significant excess over background is observed the upper limit to the flux is established. Then, the corresponding upper limit at $90 \%$ confidence level of the flux from the source is [15]:

$$
L\left(n_{o b s}, n_{b}\right)=\Phi \frac{\mu_{90}\left(n_{o b s}, n_{b}\right)}{n_{s}}=\frac{\mu_{90}\left(n_{o b s}, n_{b}\right)}{A_{E f f} \cdot T}
$$

As for the 2008-2012 analysis [3], a binned method is used in order to optimize the sensitivities of ANTARES in the search for neutrinos from cosmic ray interactions in the Sun. The Model Rejection Factor (MRF) is used to optimize the angular distance to the sources $\left(0 \leq\right.$ angle $\left.\leq 3^{\circ}\right)$ and the track quality cut parameter $0 \leq \chi^{2} \leq 2$ for events detected with more than one line, so both zenith and azimuth of neutrino direction could be determined with good accuracy.

Table 2 summarises the results of the study. The selection parameters that optimise the sensitivity are shown. It is also presented the effective area times the live time of the detector for these parameters and the observed and expected background events. Finally, the SAv flux sensitivity and upper limit obtained are shown.

\begin{tabular}{ccccccc}
\hline \multicolumn{2}{c}{ Parameters } & $A_{\text {eff }} \cdot T$ & \multicolumn{2}{c}{ Events } & Sensitivity & Upper flux Limit \\
$\chi^{2}$ & Angle $\left[{ }^{\circ}\right]$ & {$\left[\mathrm{m}^{2} \cdot \mathrm{d}\right]$} & $n_{\text {obs }}$ & $n_{b}$ & {$\left[\mathrm{~km}^{-2} \cdot \mathrm{y}^{-1}\right] \cdot 10^{12}$} & {$\left[\mathrm{~km}^{-2} \cdot \mathrm{y}^{-1}\right] \cdot 10^{12}$} \\
\hline 1.4 & 2.1 & $3.4 \cdot 10^{-4}$ & 1 & 1.8 & 3.9 & 3.5 \\
\hline
\end{tabular}

Table 2. Values of the parameters that optimise the sensitivity, the effective area times the live time, the observed and expected background events, and the SAv flux sensitivity and upper limit obtained.

\section{Solar neutrino floors for ANTARES}

We have derived the floor for Secluded Dark Mather (SDM) searches in the Sun with the ANTARES detector due to interactions of cosmic rays in the atmosphere of the Sun. Particularly, the case when two DM particles annihilates to meta-stable mediators which, in turn, decay into neutrino plus antineutrino [16] [4] [17] has been selected as example because of the enhanced signal in high-energy neutrino telescopes. For this purpose, we have to reinterpret the flux of SAv in terms of flux for the SDM detection studies. A simplified approach to the problem is to weight the original neutrino flux $\phi_{A S_{v}}$ with the effective areas for SDM and SAv, as follows:

$$
\phi_{v}=\phi_{A S_{v}} \cdot \frac{A_{E f f, A S_{v}}}{A_{E f f, S D M}}
$$


From the optimization of the event selection criteria for SDM searches with ANTARES [4], we obtained the best sensitivities from neutrino fluxes using the Model Rejection Factor (MRF) method [15], as well as the effective areas $A_{E f f, S D M}$. With these best sensitivities we have the best quality and angular cuts. In addition, from the present study we obtain the corresponding effective areas $A_{E f f, A S_{v}}$ for the resulting quality and angular cuts for SDM searches. We present the results of the floor for the case of mediator that decays directly into neutrinos, in which the neutrino signal is enhanced, and thus the one that first will reach the floor. This happens in the situation in which the mediator lifetime is long enough, so that the absorption of neutrinos in the Sun becomes negligible, but not so long that the mediator decays before reaching the Earth. In this scenario, for long-lived mediators $\left(L>10^{5} \mathrm{~km}\right.$ ), the relationship between $\Gamma$ and $\phi_{\nu}$ is [4]:

$$
\Gamma=\frac{4 \pi D^{2} \phi_{v}}{\frac{4}{3}\left(1-e^{-D / L}\right)}
$$

where D is the distance between the Sun and the Earth and L is the mediator's decay length, $L=\gamma c \tau$, i.e. the product of the mediator's lifetime, $\tau$, the speed of light, $c$, and the relativistic boost factor $\gamma$. The limits on the DM-proton cross sections have been derived assuming that there is equilibrium of the DM population in the Sun, and the same approximations shown in [4] [18]. Figure 4 shows the neutrino floor for SDM in the case of mediator decay into neutrino for a decay length of $2.8 \cdot 10^{7} \mathrm{~km}$. Here, we only consider Spin-Dependent (SD) cross sections, as direct detection experiments are more efficient for testing the Spin-Independent cross sections. The floor for ANTARES is more than two orders of magnitude below the current limit. IceCube limit from [17] is closer to the neutrino floor, although this should be taken with caution since the floor does depend in the detector as well, and the floor presented here is for ANTARES.

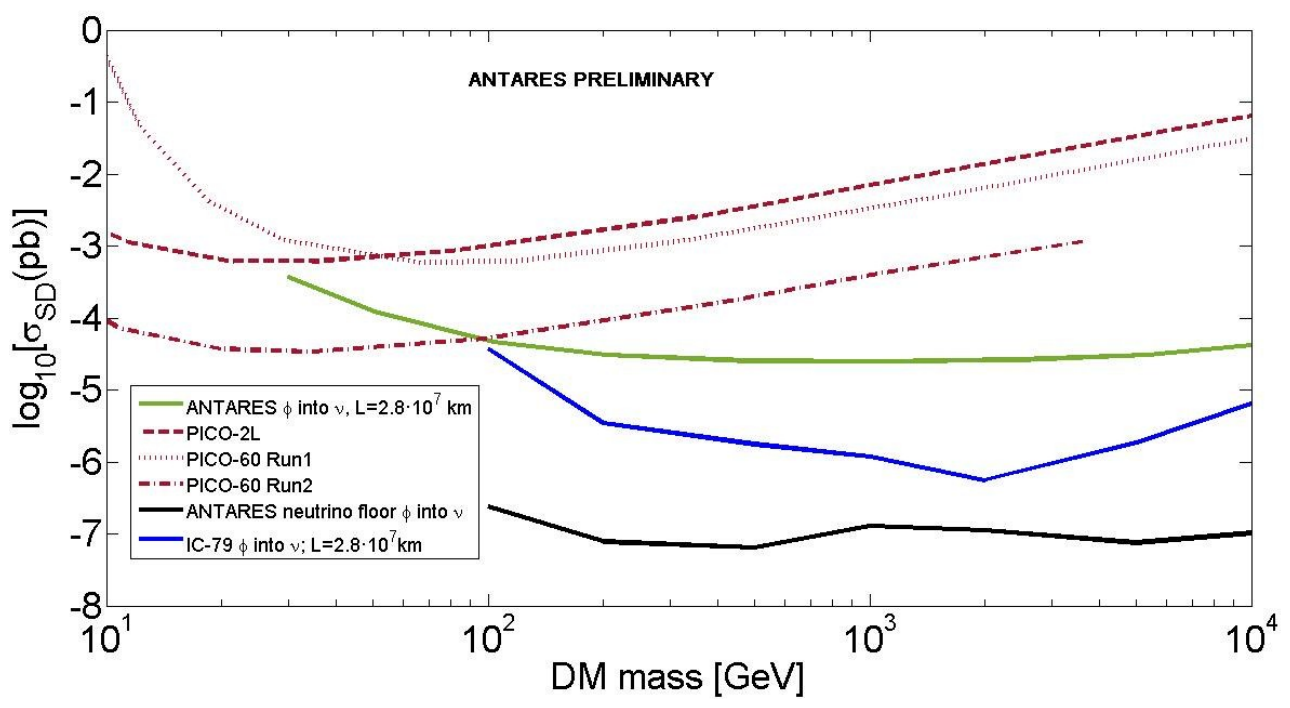

Figure 4. ANTARES neutrino floor due to SAv for the SDM model in which the mediator decays directly into neutrinos. It is compared with the limits from ANTARES [4] and IceCube-79 [19] [17] and the limits from the direct detection experiments PICO [20] [21] [22] [23]. 


\section{Conclusions}

We have obtained the sensitivity of ANTARES for SAv flux, and derived an upper limit to this flux. We have also studied the SAv as a background to the signal from DM annihilation in the Sun. Particularly for SDM that annihilates into meta-stable mediators that decay into neutrinos. For this SDM case, in which we expect the largest flux, the floor due to SAv is still more than one order of magnitude below the current ANTARES upper limit. Anyway, the SAv flux will be an essentially irreducible background for neutrino searches from DM annihilation in the Sun, and thus, this background should be studied as well for the next generation of neutrino telescopes, such as KM3NeT [24].

\section{Acknowledgements}

We acknowledge the financial support of Plan Estatal de Investigación, ref. FPA201565150-C3-1-P and ref. FPA2015-65150-C3-2-P (MINECO/FEDER), Consolider MultiDark CSD2009-00064 (MINECO) and of the Generalitat Valenciana, Grant PrometeoII/2014/079.

\section{References}

[1] M. Ageron et al., «ANTARES: The first undersea neutrino telescope,» Nuclear Instruments and Methods in Physics Research A, vol. 656, pp. 11-38, 2011.

[2] S. Adrián-Martínez et al., «First results on dark matter annihilation in the Sun using the ANTARES neutrino telescope,» Journal of Cosmology and Astroparticle Physics, 2013.

[3] S. Adrián-Martínez et al., «Limits on dark matter annihilation in the sun using the ANTARES neutrino telescope,» Physics Letters B, vol. 759, pp. 69-74, 2016.

[4] S. Adrián-Martínez et al., «A search for Secluded Dark Matter in the Sun with the ANTARES neutrino telescope,» Journal of Cosmology and Astroparticle Physics, vol. 2016, nº 5, 2016.

[5] C. Hettlage, K. Mannheim and J. Learned, «The Sun as a high-energy neutrino source,» Astroparticles Physics, vol. 13, pp. 45-50, 2000.

[6] J. Edsjö et al., «Neutrinos from cosmic ray interactions in the Sun,» arxiv 1704.02892, 2017.

[7] C. Argüelles et al., «Solar Atmospheric Neutrinos and the Sensitivity Floor for Solar Dark Matter Annihilation Searches,» arXiv:1703.07798v1.

[8] K. Ng et al., «Solar Atmospheric Neutrinos: A New Neutrino Floor for Dark Matter Searches,» arXiv:1703.10280v1.

[9] M. Masip, «High energy neutrinos from the Sun,» arXiv:1706.01290 [hep-ph].

[10] G. Ingelman and M. Thunman, «High Energy Neutrino Production by Cosmic Ray Interactions in the Sun,» Physical Review D, vol. 54, pp. 4385-4392, 1996.

[11] J. Vernazza, E. Avrett and R. Loeser, «Structure of the solar chromosphere. III - Models of the EUV brightness components of the quiet-sun,» Astrophysical Journal Supplement Series, vol. 45, pp. 635$725,1981$.

[12] J. Fontenla, K. Balasubramaniam and J. Harder, «Semiempirical models of the solar atmoshpere. The quiet sun low chromosphere at moderate resolution.,» Astrophysical Jouranl, vol. 667, n 2 , p. 1243, 2007.

[13] J. Christensen, Lecture Notes on Stellar Structure and Evolution, Institut for Fysik og Astronomi, Aarhus Universitet, 2008.

[14] G. Feldman and R. Cousins, «Unified approach to the classical statistical analysis of small signals,» Physical Review D, vol. 57, p. 3873, 1998.

[15] G. Hill and K. Rawlins, «Unbiased cut selection for optimal upper limits in neutrino detectors: the model rejection potential technique,» Astroparticle Physics, vol. 19, nº 3, pp. 393-402, 2003. 
[16] N. F. Bell and . K. Petraki, «Enhanced neutrino signals from dark matter annihilation in the Sun via metastable mediators,» JCAP, vol. 04, nº 003, 2011.

[17] M. Ardid et al., «Constraining Secluded Dark Matter models with the public data from the 79-string IceCube search for dark matter in the Sun,» Journal of Cosmology and Astroparticle Physics, vol. 2017, 2017.

[18] A. Ibarra, M. Totzauer and S. Wild, «Higher order dark matter annihilations in the Sun and implications for IceCube,» JCAP, vol. 04, p. 012, 2014.

[19] M. Aartsen et al., «Search for dark matter annihilations in the Sun with the 79-string IceCube detector,» Physical Review Letters, vol. 110, p. 131302, 2013.

[20] C. Amole et al., «Dark Matter Search Results from the PICO-2L C3F8 Bubble Chamber,» Physical Review Letters, vol. 114, 2015.

[21] C. Amole et al., «Dark matter search results from the PICO-60 CF3I bubble chamber,» Physical Review D, vol. 93, p. 050214, 2016.

[22] C. Amole et al., «Improved dark matter search results from PICO-2L Run 2,» Physical Review D, vol. 93, p. 061101(R), 2016.

[23] C. Amole et al., «Dark Matter Search Results from the PICO-60 C3F8 Bubble Chamber,» Phys. Rev. Lett., vol. 118, $\mathrm{n}^{\mathrm{o}}$ 251301, 2017.

[24] S. Adrián-Martínez et al., «Letter of intent for KM3NeT 2.0,» J. Phys. G, vol. 43, nº 084001, 2016. 